\title{
Comparative Evaluation of DWT and DT-CWT for Image Fusion and De-noising
}

\author{
Rudra Pratap Singh \\ Chauhan \\ Research Scholar \\ UTU, Dehradun, (U.K.), India
}

\author{
Rajiva Dwivedi, Phd. \\ Bharat Institute of Technology, \\ Meerut, (U.P.)
}

\author{
Sandeep Negi \\ Uttaranchal Institute of \\ Technology, Dehradun, (U.K.)
}

\begin{abstract}
In various real life applications such as remote sensing and medical image diagnosis, image fusion plays imperative role and it is more popular for image processing applications. Because of inadequate nature of practical imaging systems the capture images or acquired images are corrupted from various noise hence fusion of image is an integrated approach where reduction of noise and retaining the original features of image is essential. Image fusion is the process of extracting meaningful visual information from two or more images and combining them to form one fused image. Discrete Wavelet Transform (DWT) has a wide range of application in fusion of noise images. Previously, real valued wavelet transforms have been used for image fusion. Although this technique has provided improvements over more inhabitant methods, this transform suffers from the shift variance and lack of directionality associated with its wavelet bases. These problems have been overcome by the use of a reversible and discrete complex wavelet transform (the Dual Tree Complex Wavelet Transform DT-CWT). This paper therefore introduces an alternative structure such as DT-CWT that is more flexible, highly directional and shift invariant which outperforms the conventional method in terms of PSNR and image quality improvement.
\end{abstract}

\section{General Terms}

Image, Wavelet, Fusion of image. Image fusion rule, Denoising.

\section{Keywords}

Wavelet transform, Discrete Wavelet Transform (DWT), Dual-Tree Complex Wavelet Transform (DT-CWT), Image Fusion..

\section{INTRODUCTION}

The successful fusion of images acquired from diverse instruments is of great importance in many applications, such as medical imaging, microscopic imaging, remote sensing, computer vision, and robotics. Image fusion can be defined as the processes by which several images, or some of their features, are combined together to form a single image. Image fusion can be performed at different levels of the information representation. Four different levels can be distinguished according to signal[1], pixel, feature and symbolic levels. To date, the results of image fusion in areas such as remote sensing and medical imaging are primarily intended for presentation to a human observer for easier and enhanced interpretation. Therefore, the perception of the fused image is of paramount importance when evaluating different fusion schemes. When fusion is done at pixel level the input images are combined without any pre-processing. Pixel level fusion algorithms vary from very simple, e.g. image averaging, to very complex, e.g. principal component analysis, pyramid based image fusion and wavelet transform fusion. Since the DWT has some limitation such as less directional selectivity, shift-invariance, aliasing, oscillation of wavelet coefficients and required higher computational cost because of highly redundant representation. These limitations of DWT are overcome by Dual Tree Complex Wavelet Transform(DTCWT) up to the great extent. Here in this literature we have proposed a novel approach of DT-CWT based image fusion method. Even though it has complexity to implement but it gives best results than the Discrete Wavelet Transform based image fusion method.

\section{WAVELET TRANSFORM FUSION}

The basic idea of all multi-resolution fusion schemes is motivated by the human visual system being primarily sensitive to local contrast changes, e.g. the edges or corners. In the case of wavelet transform fusion all respective wavelet coefficients from the input images are combined using the fusion rule. Since wavelet coefficients having large absolute values contain the information about the salient feature of the images such as edges and lines. Many wavelet based techniques for fusion of 2-D images have been described in the literature $[3,4,5,6]$. In all wavelet based image fusion schemes the wavelet transforms $\mathrm{W}$ of the two registered input images $\mathrm{I}_{1}(\mathrm{x}, \mathrm{y})$ and $\mathrm{I}_{2}(\mathrm{x}, \mathrm{y})$ are computed and these transforms are combined using some kind of fusion rule $\Phi$, Figure(1). Then, the inverse wavelet transform $\mathrm{W}^{-1}$ is computed and the fused image $\mathrm{I}(\mathrm{x}, \mathrm{y})$ is reconstructed:

$$
\mathrm{I}(\mathrm{x}, \mathrm{y})=\mathrm{W}^{-1}\left(\Phi\left(\mathrm{W}\left(\mathrm{I}_{1}(\mathrm{x}, \mathrm{y})\right), \mathrm{W}\left(\mathrm{I}_{2}(\mathrm{x}, \mathrm{y})\right)\right)\right)
$$

Wavelet transform fusion schemes offer several advantages over pyramid based fusion schemes when it comes to image fusion the wavelet transform provides high directional information while the pyramid representation does not introduce any spatial orientation in the decomposition process. 

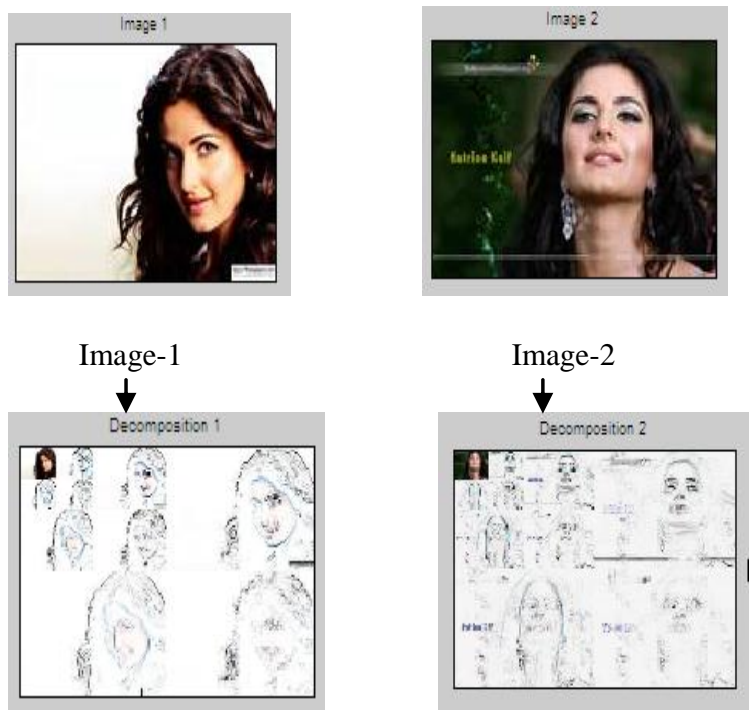

Wavelet Decomposition

Wavelet Decomposition of Image-1 at Level-3

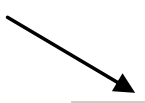
of Image-2 at Level-3

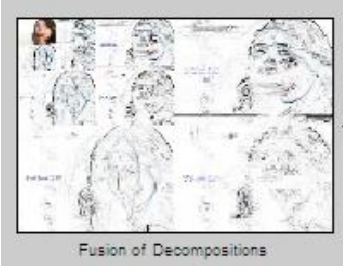

Fused Wavelet Coefficients of Image- 1 and Image-2 at level-3
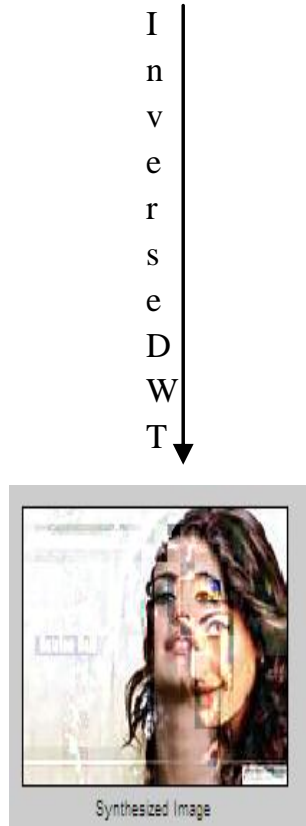

Synthesized or Fused Image

Fig: 1: Fusion of Two Images using Wavelet Transforms
[Li et al., 1995], in pyramid based image fusion, the fused images often contain blocking effects in the regions where the input images are significantly different.. Images generated by wavelet image fusion have better signal-to- noise ratios(SNR) than images generated by pyramid image fusion when the same fusion rules are used [Wilson et al. 1995] [1,6].

\section{THE DISCRETE WAVELET TRANSFORM}

The discrete wavelet transform (DWT) is a spatial-frequency decomposition that provides a flexible multi-resolution analysis of an image. In one dimension the aim of the wavelet transform is to represent the signal as a superposition of wavelets. If a discrete signal is represented by $f(t)$, its wavelet decomposition is then

$$
f(t)=\sum_{m, n} C_{m, n} \psi_{m, n}(t)
$$

Where $m$ and $n$ are integers. This ensures that the signal is decomposed into normalized wavelets at octave scales. For an iterated wavelet transform additional coefficients $a_{m, n}$ are required at each scale. At each $\mathrm{a}_{\mathrm{m}, \mathrm{n}}$ and $\mathrm{a}_{\mathrm{m}-1, \mathrm{n}}$ describe the approximations of the function $\mathrm{f}$ at resolution $2_{\mathrm{m}}$ and at the coarser resolution $2_{\mathrm{m}-1}$ respectively, while the coefficients $\mathrm{c}_{\mathrm{m}, \mathrm{n}}$ describe the difference between one approximation and the other. In order to obtain the coefficients $c_{m, n}$ and $a_{m, n}$ at each scale and position, a scaling function is needed that is similarly defined to equation(3). The convolution of the scaling function with the signal is implemented at each scale through the iterative filtering of the signal with a low pass FIR filter $\mathrm{h}_{\mathrm{n}}$. The approximation coefficients $\mathrm{a}_{\mathrm{m}, \mathrm{n}}$ at each scale can be obtained using the following recursive relation:

$\boldsymbol{a}_{m, n}=\sum_{k} \square_{2 n-k} a_{m-1, k}$

Where the top level $a_{0, n}$ is the sampled signal itself. In addition, by using a related high pass FIR filter $g_{n}$ the wavelet coefficients can be obtained by:

$$
c_{m, n}=\sum_{k} g_{2 n-k} a_{m-1, k}
$$

To reconstruct the original signal the analysis filters can be selected from a bi-orthogonal set which have a related set of synthesis filters. These synthesis filters $\widetilde{\square}$ and $\widetilde{\boldsymbol{g}}$ can be used to perfectly reconstruct the signal using the reconstruction formula:

$$
\begin{aligned}
& \boldsymbol{a}_{m-1, l}(f)=\sum_{n}\left[\widetilde{h}_{2 n-1} a_{m, n}(f)+\right. \\
& \left.\widetilde{g}_{2 n-1} c_{m, n}(f)\right]
\end{aligned}
$$

Equations (4) and (5) are implemented by filtering and subsequent down sampling. Conversely equation (6) is implemented by an initial up sampling and a subsequent filtering. A single stage wavelet synthesis and analysis in one dimension is shown in figure(2). 


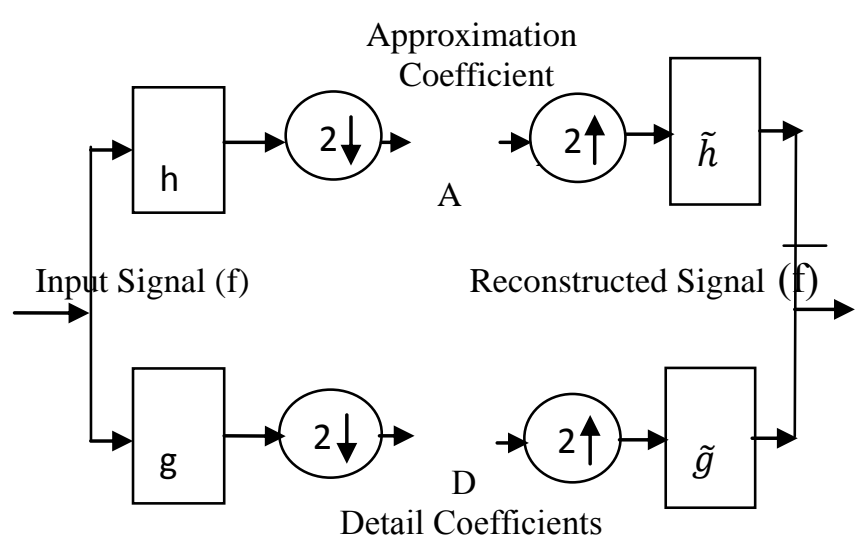

Fig. 2: One Dimensional Wavelet Analysis and Synthesis Filters

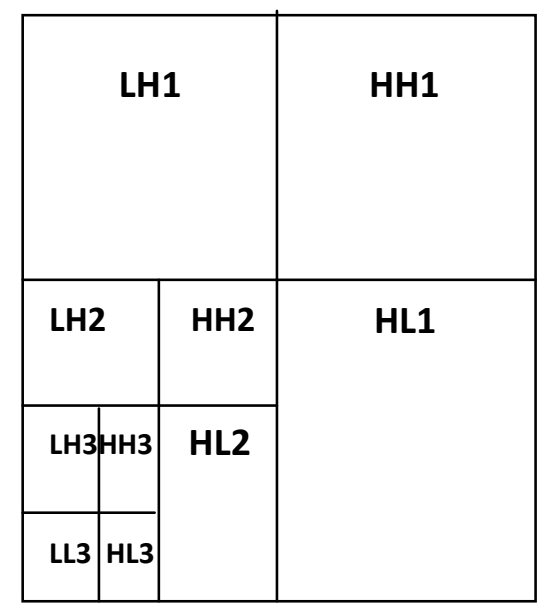

Fig. 3: Wavelet Decomposition at Level-3

The fusion process of two images using the DWT is shown in. figure (4). The two images used were from a multi-focus set, i.e. two registered images of same scene each with a different camera focus. This figure shows that the coefficients of each transform have significantly different magnitudes within the regions of different focus. A simple "maximum selection" was used to generate the combined coefficient map. This effectively retains the coefficients of "in focus" regions within the image. This inverse wavelet transform is then applied to the combined coefficient map to produce the fused image which in this case shown an image retaining the focus from the two input images.

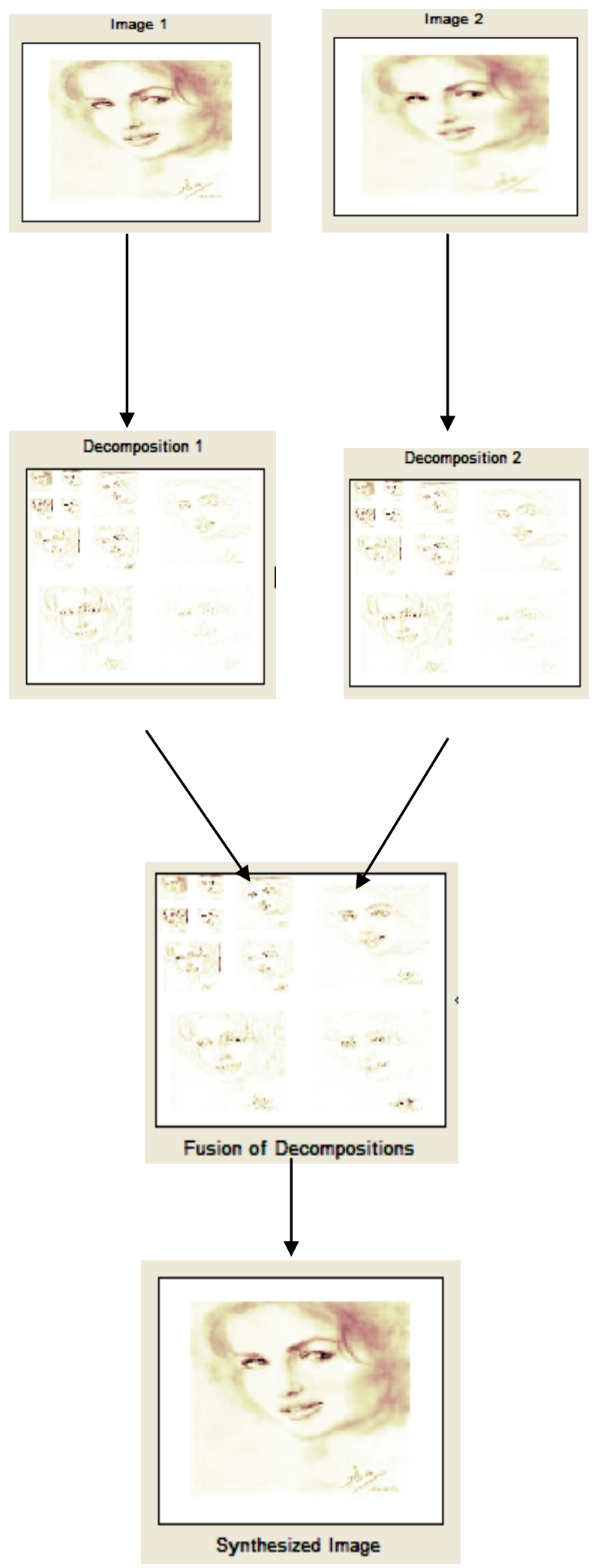

Fig: 4: Image fusion process using the DWT and two registered multi-focus Katrina Kaif images. 


\section{THE DUAL-TREE COMPLEX WAVELET TRANSFORM}

The Dual-tree Complex wavelet transform (DT-CWT)[2,3,10] is complex valued extension of the standard wavelet. Complex transform uses complex valued filtering that decomposes the image into real and imaginary parts in transform domain. The real and imaginary coefficients are used to compute magnitude and phase information. The prime motivation for producing the dual-tree complex wavelet transform was shift invariance. In a normal wavelet decomposition small shifts of the input signal are able to move energy between output sub-bands. Shift invariance can also be achieved in DWT by doubling the sampling rate. This is effected in the DT-CWT by eliminating the down sampling by 2 after first level filter. Two fully decimated trees are then produced by down sampling, effected by taking first even and then odd samples after the first level of filters. To get uniform intervals between the two trees samples, the subsequent filters need half a sample different delay in one tree. Application to image can be achieved by separable complex filtering in two dimensions.

The real 2-D dual-tree DWT of an image $\mathrm{x}$ is implemented using two critically-sampled separable 2-D DWTs in parallel. Then for each pair of sub-bands we take the sum and difference. The complex 2-D DT-DWT also gives rise to wavelets in six distinct directions. The complex 2-D dual-tree is implemented as four critically-sampled separable 2-D DWTs operating in parallel as shown in figure (5). 2-D structure needs four trees for analysis and synthesis. The pair of conjugate filters applied to two dimensional image $(x, y)$ can be expressed as:

$$
\left(h_{x}+j g_{x}\right)\left(h_{y}+j g_{y}\right)=\left(h_{x} h_{y}-g_{x} g_{y}\right)+j\left(h_{x} g_{y}+g_{x} h_{y}\right)
$$

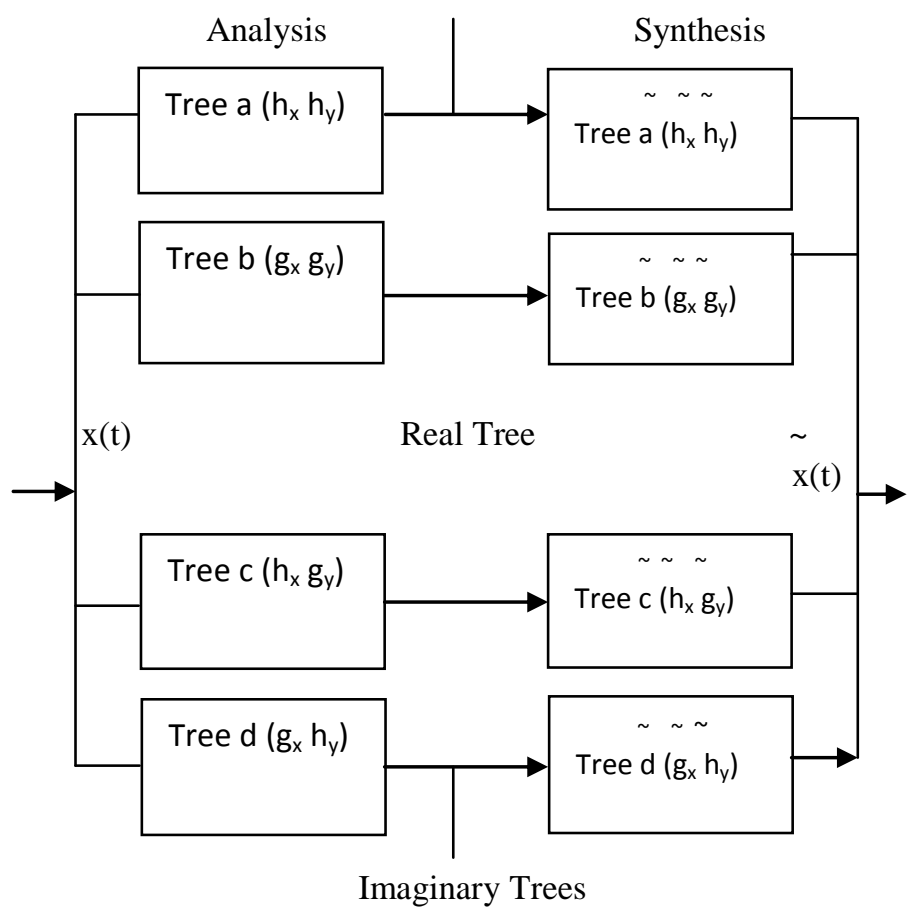

Fig. 5: Filter bank structure for 2-D DT-DWT
The complex wavelets are able to distinguish between positive and negative the diagonal sub-bands can be distinguished and horizontal and vertical sub-bands are divided giving six distinct sub-bands in each scale at orientations $\pm 15^{\circ}, \pm 45^{0}$, $\pm 75^{\circ}$. The oriented and scale dependent sub-bands are visualized spatially in figure (6).

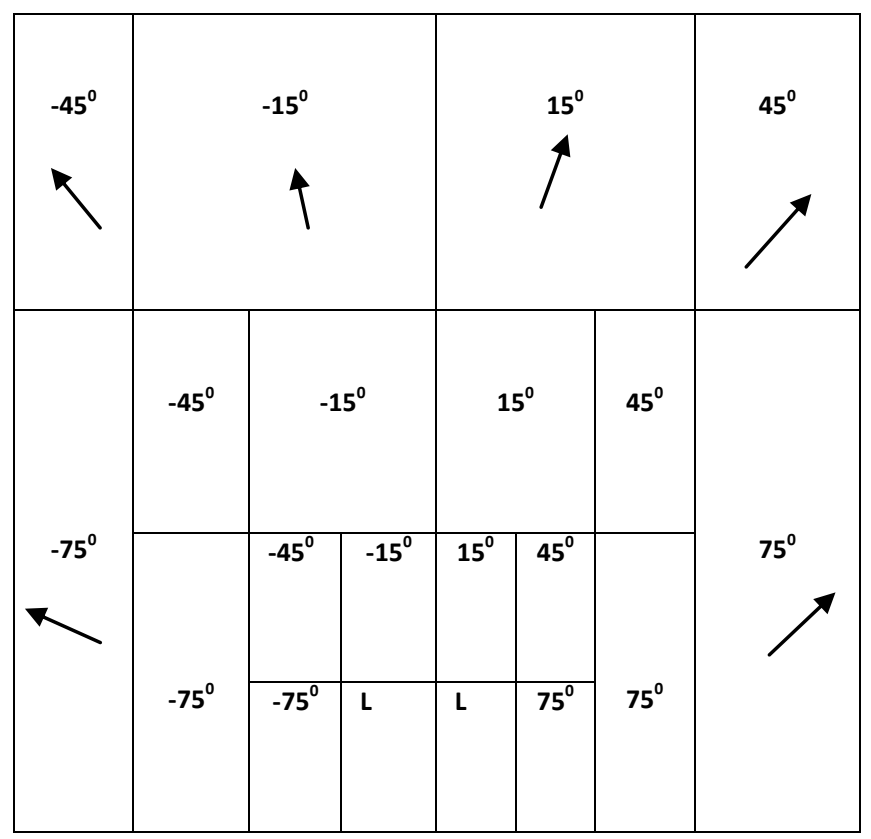

Fig 6: Complex Wavelet Transform Scale Orientation labeled sub- bands

The DWT have three sub-bands in $0^{\circ}, 45^{\circ}$ and $90^{\circ}$ directions only but DT-CWT having six sub-bands in $\pm 15^{\circ}, \pm 45^{\circ}$ and $\pm 75^{\circ}$, thus DT-CWT improves the directional selectivity which is the prime concern in the application like image fusion.

\section{IMPLEMENTATION OF FUSION RULE}

Three previously developed fusion rule schemes were implemented using discrete wavelet transform based image fusion:

- Maximum Selection (MS) Scheme: This MS scheme picks the coefficient in each sub-band with the largest magnitude.

- Weighted Average (WA) Scheme: In this scheme a normalized correlation between the two images sub-bands over a small local area. The resultant coefficient for reconstruction is calculated from this measure via a weighted average of the two images coefficients.

- Window based Verification (WBV) Scheme: It creates a binary decision map to choose between each pair of coefficients using a majority filter[1]. 


\section{RESULT}

The performance of DWT fusion method and DT-CWT fusion methods are compared by considering two different color images of Catherine 1(Left corrupted) and Catherine 2 (Right Corrupted) . The Synthesized image after DWT fusion method and DT-CWT based fusion methods are a shown in figures (7) and (8) respectively. It has been observed from figure(8) that wavelet transform is an essential tool for image fusion and de-noising. Due to improved directive and shift invariant properties of DT-CWT fusion method outperforms the DWT fusion method .
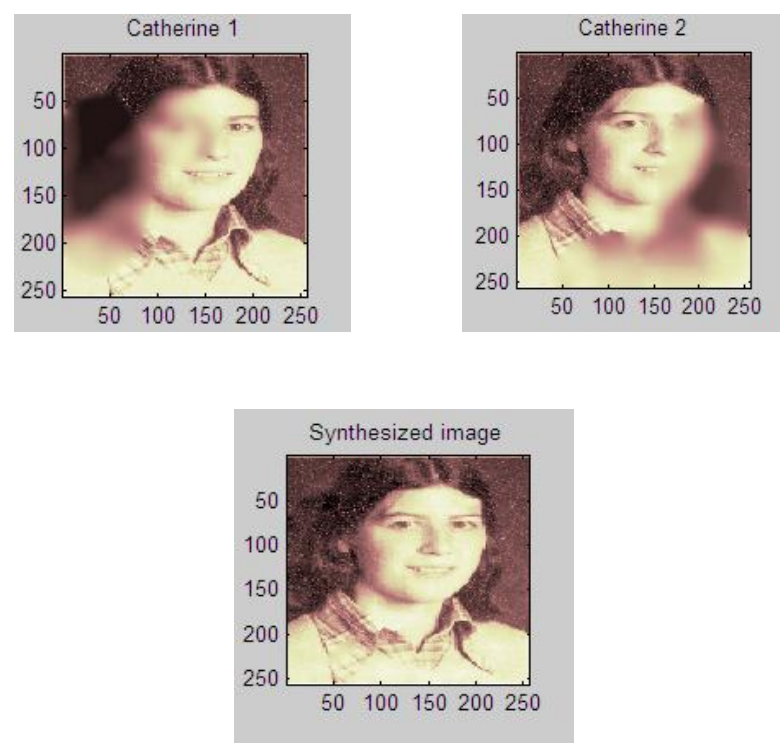

Fig. 7: Image Fusion and De-noised Image Using DWT
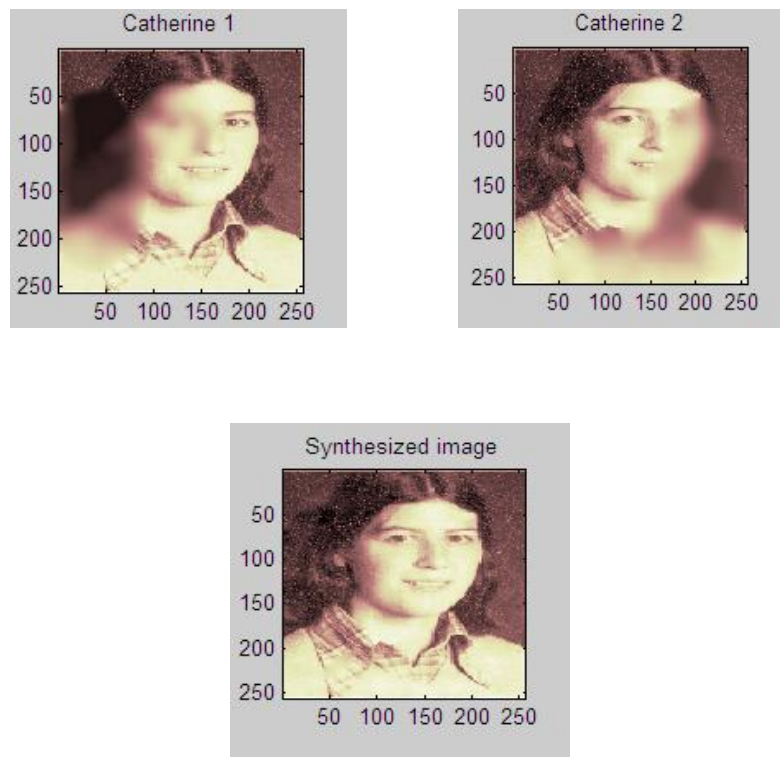

Fig. 8: Image Fusion and De-noised Image Using DTCWT
Comparison and performance evaluation of different test images using DWT and DT-CWT is tabulated below. Table 1 shows the Peak Signal-to-Noise-Ratio (PSNR) and Normalized Cross Correlation (NCC) of different images using DWT and DT-CWT.

Table1: Comparison evaluation between DWT and DT-CWT for three different images.

\begin{tabular}{|c|l|c|c|c|c|c|}
\hline Images & \multicolumn{2}{|c|}{$\begin{array}{c}\text { Katrina Kaif } \\
\text { Image }\end{array}$} & \multicolumn{2}{c|}{$\begin{array}{c}\text { Catherine } \\
\text { Image }\end{array}$} & \multicolumn{2}{c|}{ Mask Image } \\
\hline & PSNR & NCC & PSNR & NCC & PSNR & NCC \\
\hline DWT & $\mathbf{2 0 . 6 9}$ & $\mathbf{0 . 8 6}$ & $\mathbf{1 8 . 6 4}$ & $\mathbf{0 . 8 5}$ & $\mathbf{2 0 . 3 9}$ & $\mathbf{0 . 8 7}$ \\
\hline DT-CWT & $\mathbf{3 2 . 5 4}$ & $\mathbf{0 . 9 6}$ & $\mathbf{3 0 . 4 5}$ & $\mathbf{0 . 9 7}$ & $\mathbf{3 1 . 1 7}$ & $\mathbf{0 . 9 5}$ \\
\hline
\end{tabular}

\section{Comparative Analysis of DWT and DT-CWT}

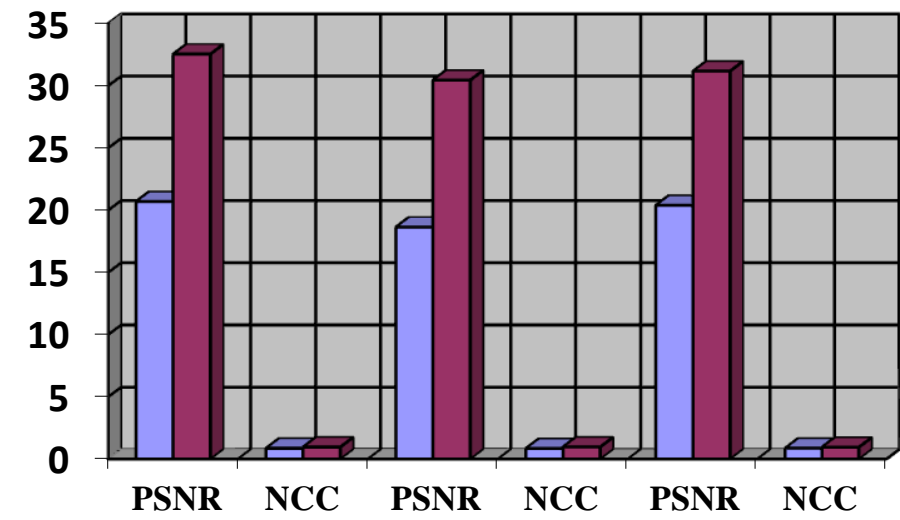

\section{口DWT 口DT-CWT}

Fig 9: Comparative analysis of DWT and DT-CWT of three different images

\section{CONCLUSION}

The DT-CWT fusion technique of noisy Catherine 1 and Catherine 2 provides better results than the DWT fusion technique. The DT-CWT based fusion method is able to retain important edge information without significant humming artifacts. DT-CWT provides increased shift-invariance and orientation selectivity when compared to the DWT. This is demonstrated and furnished in table 1 shown above. The above figure 9 shows the analysis and performance of DWT and DTCWT for three test images of Katrina Kaif, Mask image and Catherine image.

\section{ACKNOWLEDGMENTS}

We are thankful for N. G. Kingsbury for providing us the necessary code for image fusion and de-noising without that it is not possible for us to do research in this field. 


\section{REFERENCES}

[1] \{Chipman et al. 1995\} Chipman, L. J., Orr, T. M., and Lewis, L. N. (1995). Wavelets and image fusion. In Proceedings IEEE International Conference on Image Processing, Washigton D. C., volume 3, pages 248-251. IEEE.

[2] N. G. Kingsbury, "The dual-tree complex wavelet transform with improved orthogonality and symmetry properties", IEEE international Conference on Image processing, pages 375-378, September 2000.

[3] N. G. Kingsbury, "The dual-tree complex wavelet transform: a new technique for shift invariance and directional filters, IEEE Digital Signal Processing Workshop, 1998.

[4] S. M. Mahbubur Rahman, M. Omair Ahmad and M.N.S Swamy, "Constant-based fusion of noisy image using discrete wavelet transform", IET Image Process, 2010, Vol. 4 Iss.5, pp. 374-384 doi:10. 1049/ iet-ipr. 20009.0163.

[5] Koren, I. and Laine, A. (1998). A discrete dyadic wavelet transform for multidimensional feature analysis. In Akay, M, editor, Time Frequency and Wavelets in Biomedical Signal Processing, pages 425-449. IEEE Press.

[6] Koren, I. and Laine, A. and Tylor, F. (1995). Image fusion using steerable dyadic wavelet transforms. In proceedings IEEE International Conference on Image Processing, Washington D.C., pages 232235. IEEE.

[7] Resources for research in image fusion :[Online], http://www.imagefusion.org/

[8] The Math works, 'Wavelet Toolbox (ver 5) User's guide', 2007, URL: www.mathworks.com

[9] H.B. Mitchell. Image Fusion theories, techniques, and applications", ISBN 978-642-11215-7, Springer-Verlag Berlin Heidelberg, 2010.

[10] Kingsbury, N.G.(2000) "A dual-tree complex wavelet transform with improved orthogonality and symmetry properties. Proc. IEEE Conf. on Image Processing, Vancouver, September 11-13, 2000, (paper 1429).

[11] Nikolov, S. G., Bull, D. R., Canagarajah, C. N., Halliwell, M. and Wells, P.N.T. (2000), 2-D image fusion by multiscale edge graph combination. In $3^{\text {rd }}$ International Conference on Information Fusion (Fusion 2000), Paris, France, 10-13 July, volume I, pages MoD3-16-22. International Society of Information Fusion (ISIF).

[12] Zhang, Z. and Blum, R. (1999). A categorization of multiscale-decomposition-based image fusion schemes with a performance study for a digital camera application. Proceedings of the IEEE. Pages 1315-1328. 